\title{
Comparación de Algoritmos Lineales y no Lineales para la Detección del Desacoplamiento Cardiorrespiratorio en Ratas Endotoxémicas
}

\section{Comparison of Linear and Nonlinear Algorithms for Detection of Cardiorespiratory Uncoupling in Endotoxemic Rats}

\author{
I. M. Flores-Duarte', A. C. Pliego-Carrillo', C. I. Ledesma-Ramírez', J. C. Echeverría-Arjonilla², M. A. Peña-Castillo², \\ G. Pacheco-López ${ }^{3}$, J. J. Reyes-Lagos ${ }^{1}$ \\ 1Facultad de Medicina, Universidad Autónoma del Estado México \\ ²Departamento de Ingeniería Eléctrica, Universidad Autónoma Metropolitana, Unidad Iztapalapa \\ 32Departamento de Ciencias de la Salud, Universidad Autónoma Metropolitana, Unidad Lerma
}

\section{RESUMEN}

En estudios previos se ha demostrado que la endotoxemia inducida por lipopolisacárido (LPS) produce un desacoplamiento cardiorrespiratorio (CRP) debido a los efectos fisiológicos de la inflamación sistémica. Adicionalmente se sabe que la oxitocina tiene efectos antiinflamatorios y propiedades cardioprotectoras; sin embargo, se desconoce si ésta modifica el acoplamiento CRP. El objetivo del presente estudio fue comparar diferentes métodos matemáticos lineales y no lineales para la detección del desacoplamiento cardiorrespiratorio entre series de tiempo cardiacas y respiratorias. Se estudiaron series de tiempo R-R obtenidas de electrocardiogramas de grupos de roedores macho a los cuales se les administró solución salina o vehículo (V); lipopolisacárido (LPS); oxitocina (O) y LPS + oxitocina (LPS+Ox). Las series R-R y respiratorias derivadas del electrocardiograma (EDR) se analizaron en conjunto para cuantificar su grado de acoplamiento a través de las técnicas de correlación cruzada; entropía muestral cruzada; entropía condicional; información mutua; e información mutua de Rényi para los cuatro grupos. Se observó que la oxitocina no parece favorecer el acoplamiento CRP durante la endotoxemia inducida por LPS. Finalmente, se encontró que la entropía muestral cruzada y la entropía condicional presentaron las mayores diferencias estadísticas para identificar el desacoplamiento CRP producido por el LPS.

PALABRAS CLAVE: endotoxemia; oxitocina; acoplamiento cardiorrespiratorio; respiración derivada del electrocardiograma 


\section{ABSTRACT}

Previous studies have shown that LPS-induced endotoxemia causes a cardiorespiratory (CRP) uncoupling owing to the physiological effects of systemic inflammation. Also, it is known that oxytocin has anti-inflammatory effects and cardioprotective properties; however, it is unknown whether it can modify the CRP coupling. This study aimed to compare different linear and nonlinear mathematics methods for the detection of cardiorespiratory uncoupling between cardiac and respiratory time series. The R-R time series of electrocardiograms of male rodents that were administered with saline solution (V); lipopolysaccharide (LPS); oxytocin (O) and LPS + oxytocin (LPS + Ox) were studied. We tested the R-R and respiratory series derived from the electrocardiogram (EDR) for the four groups to quantify the degree of coupling with cross-correlation; cross sample entropy; conditional entropy; mutual information; and Rényi's mutual information. We found that oxytocin does not seem to favor the CRP coupling during endotoxemia induced by LPS. Finally, we observed that the cross-sample entropy and the conditional entropy presented the highest statistical differences to identify the CRP uncoupling produced by LPS.

KEYWORDS: endotoxemic; oxytocin; cardiorespiratory coupling; ECG derived respiration

\section{Correspondencia}

DESTINATARIO: José Javier Reyes Lagos

INSTITUCIÓN: Facultad de Medicina, Universidad

Autónoma del Estado México

DIRECCIÓN: Av. Paseo Tolloacan S/N, esquina Jesús

Carranza, Col. Moderna de la Cruz, C. P. 50180, Toluca

de Lerdo, Estado de México, México

CORREO ELECTRÓNICO: jjreyesl@uaemex.mx

\section{Fecha de recepción:}

3 abril de 2019

Fecha de aceptación:

1 de julio de 2019 


\section{INTRODUCCIÓN}

En la actualidad, el estudio de la interacción de fenómenos fisiológicos en los seres vivos ha tenido avances significativos, específicamente la medición de correlaciones entre señales fisiológicas ha sido motivo de diversas investigaciones. Hallazgos importantes combinan la aplicación de métodos de análisis de series de tiempo con el registro simultáneo de una o más señales fisiológicas [1]. En particular, la interacción de fluctuaciones R-R extraída del electrocardiograma (ECG) con la actividad respiratoria, es uno de los fenómenos fisiológicos más explorados con estos métodos ${ }^{[2,3]}$.

En diversos estudios se ha investigado la existencia de un vínculo funcional entre el ritmo cardiaco y respiratorio, al que se le ha denominado acoplamiento cardiorrespiratorio (CRP) ${ }^{[4-6]}$. El acoplamiento CRP, describe las influencias de la respiración sobre la frecuencia

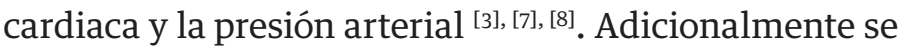
ha utilizado al acoplamiento CRP para cuantificar la relación entre el sistema cardiaco y respiratorio bajo el control del sistema nervioso central ${ }^{[9]}$.

Gholami et. al han reportado que las manifestaciones clínicas relacionadas a una exagerada reacción inflamatoria (ej. endotoxemia) se asocian con un desacoplamiento parcial de las células marcapasos del sistema nervioso autónomo (SNA), lo que origina la disminución de la variabilidad de la frecuencia cardiaca (VFC) ${ }^{[10]}$. Debido a la alta asociación entre el SNA con las células marcapasos cardiacas, la VFC no es el único parámetro fisiológico afectado, sino también lo es el ritmo respiratorio ${ }^{[10]}$.

La inyección de lipopolisacárido (LPS) causa un desacoplo en la regulación autonómica en ratas conscientes [11], dicho desacoplamiento se manifiesta como taquicardia, pérdida de la dinámica fractal de series de tiempo R-R, cambios en la temperatura corporal y comportamiento letárgico ${ }^{[11]}$. Por otro lado, la neurohormona oxitocina tiene funciones fisiológicas amplia- mente conocidas en mamíferos, Gamer et. al sugieren que tiene propiedades inmunitarias y cardioprotectoras; principalmente mediante la modulación de la actividad del sistema nervioso parasimpático ${ }^{[12]}$. Nuestros estudios previos respaldan las propiedades antiinflamatorias y cardioprotectoras de la oxitocina, puesto que una dosis baja de oxitocina periférica redujo el comportamiento de enfermedad (sickness behavior) y disminuyó la taquipnea en ratas ${ }^{[11]}$. En dichas investigaciones concluimos que la oxitocina parece restablecer las interacciones cardiorrespiratorias y el acoplamiento colinérgico cardiaco ${ }^{[11,13]}$.

Bajo este contexto, el objetivo del presente estudio fue comparar diferentes métodos matemáticos lineales y no lineales para la detección del acoplamiento cardiorrespiratorio entre series de tiempo cardiacas y respiratorias obtenidas de un modelo experimental de roedores sometidos a endotoxemia mediante la aplicación de lipopolisacárido y suministración conjunta de oxitocina exógena. Hipotetizamos que los métodos no lineales podrán identificar modificaciones en el acoplamiento CRP de una manera más apropiada que los métodos lineales.

\section{METODOLOGÍA}

\section{Base de datos de electrocardiogramas telemétricos}

Las señales de ECG utilizadas para esta investigación fueron extraídas de investigaciones previas ${ }^{[11]}$, dichos datos fueron obtenidos de ratas macho Dark Agouti implantadas con un transmisor de ECG telemétrico (frecuencia de muestreo $2000 \mathrm{~Hz}$ ) como se ha descrito previamente ${ }^{[11,13]}$.

Los datos fisiológicos corresponden a cuatro grupos a los que se les aplicó el siguiente tratamiento: 1) vehículo (V; solución salina $\mathrm{n}=7$ ); 2) oxitocina (Ox; $3 \mathrm{UI} / \mathrm{Kg}$ administrada subcutáneamente, $\mathrm{n}=8$ ); 3) lipopolisacárido (LPS; 0.1mg/Kg administración intraperitoneal, 
$\mathrm{n}=8)$; 4) LPS + Oxitocina (LPS+Ox; administración combinada de $0.1 \mathrm{mg} / \mathrm{Kg}$ LPS intraperitoneal y $3 \mathrm{UI} / \mathrm{Kg}$ oxitocina subcutánea, $\mathrm{n}=8$ ).

\section{Análisis de datos}

Se seleccionaron segmentos de ECG de cinco minutos de duración en las siete horas posteriores al tratamiento $(+7 \mathrm{~h})$, puesto que nuestros estudios previos han demostrado que los mayores desajustes autonómicos cardiacos debido a la endotoxemia se manifiestan hasta este lapso de tiempo ${ }^{[11,13]}$. Los segmentos de cinco minutos de ECG se analizaron para la detección del complejo QRS y la generación de series de tiempo $\mathrm{R}$ - $\mathrm{R}$ utilizando algoritmos propios desarrollados en el software MATLAB(The MathWorksInc., Massachusetts, Estados Unidos).

Adicionalmente, se aplicó una técnica de procesamiento de señales para obtener la respiración derivada del electrocardiograma (EDR, por sus siglas en inglés) en función del área bajo la curva del complejo QRS. El algoritmo de esta técnica se encuentra disponible en PhysioNet (www.physionet.org) y proporciona datos confiables relacionados con señales fisiológicas respiratorias ${ }^{[14]}$. La ventana móvil del algoritmo se ajustó a $0.1 \mathrm{~s}$; las series de tiempo EDR y R-R se interpolaron mediante un spline cúbico a $100 \mathrm{~Hz}$, posteriormente fueron filtradas digitalmente con un pasa altas utilizando una frecuencia de corte de $0.5 \mathrm{~Hz}$.

Para la cuantificación del acoplamiento CRP se utilizaron técnicas lineales: la correlación cruzada (X-Corr) algoritmo incorporado al software MATLAB, y no lineales: la entropía muestral cruzada (CrossSampEn), la entropía condicional (CondH), la información mutua (MI) y la información mutua de Rényi (RényiMI) provenientes de los scripts de PhysioNet y MIToolbox ${ }^{[15]}$ (Universidad de Manchester, Inglaterra) disponibles en (http://academic.adampocock.com/index.html). La descripción matemática de estos parámetros se detallará a continuación.

\section{Correlación cruzada}

La función de correlación cruzada (X-Corr) ha sido empleada para estudiar la fuerza y la dirección de la relación entre dos procesos estocásticos conjuntamente estacionarios. Considerando dos procesos conjuntamente estacionarios como $x_{t}$ y $y_{t}$, para $t=0, \pm 1, \pm 2$, ... la covarianza cruzada $\gamma_{x y}$ de orden k entre $x_{t}$ y $y_{t}$ está definida como:

$$
\gamma_{\mathrm{xy}}(\mathrm{k})=\mathrm{E}\left[\left(\mathrm{x}_{\mathrm{t}}-\mathrm{y}_{\mathrm{x}}\right)\left(\mathrm{y}_{\mathrm{t}+\mathrm{k}}-\mu_{\mathrm{y}}\right)\right]
$$

Para $\mathrm{k}=0, \pm 1, \pm 2, \ldots, \gamma_{x y}(\mathrm{k})$ es llamada la función de covarianza cruzada entre $x_{t}$ y $y_{t^{*}}$

La estandarización de $\gamma_{x y}$ (k) produce la función de correlación cruzada (ecuación 2):

$$
\mathrm{p}_{\mathrm{xy}}(\mathrm{k})=\gamma_{\mathrm{xy}}(\mathrm{k}) /\left(\sigma_{\mathrm{x}} \sigma_{\mathrm{y}}\right)
$$

Para $\mathrm{k}=0, \pm 1, \pm 2, \ldots$, donde $\sigma_{x} \mathrm{y} \sigma_{y}$ son las desviaciones estándar de los procesos $x_{t}$ y $y_{t^{*}}$.

Para valores positivos de $k, \gamma_{x y}$ describe la influencia lineal de los valores pasados de $x_{t}$ en $y_{t}$ indicando un mayor acoplamiento en las señales. Un resultado de la $X$-Corr cercano a 1 indica una interacción lineal positiva entre dos señales dado un retardo $k^{[16]}$.

\section{Entropía muestral cruzada}

La entropía muestral cruzada (Cross-SampEn) es un parámetro utilizado para medir el grado de asincronía de dos series de tiempo relacionadas, por lo que se correlaciona negativamente con un nivel de acoplamiento no lineal entre dos señales ${ }^{[17]}$. El valor de la Cross-SampEn cuantifica las interacciones entre dos series de tiempo distintas pero que interactúan entre sí (en este caso, la frecuencia cardiaca y la respiración bajo la influencia del sistema nervioso central) ${ }^{[8]}$. Cuando la asociación entre los intervalos R-R y la señal EDR es fuerte, el valor de entropía cruzada será pequeño, lo que sugeriría un alto nivel de CRP. En con- 
traste, cuando hay una asociación débil entre series, los valores de la Cross-SampEn serán mayores, lo que indicaría una sincronía relativamente baja ${ }^{[18]}$. Al calcular las entropías cruzadas, los patrones qa comparar se toman en pares de las dos series de tiempo diferentes con $N$ puntos $\{\mathrm{u}(\mathrm{i})\}$ y $\{\mathrm{v}(\mathrm{i})\}, \mathrm{i}=1, \ldots, \mathrm{N}$. De un vector de $m$ secuencias, de $u$ y de $v$, respectivamente. Los vectores se construyen de la siguiente forma:

$$
\begin{aligned}
& x_{m}(i)=[u(i), u(i+1), u(1+2) \ldots . . u(i+m-1)] \\
& y_{m}(i)=[v(i), v(i+1), v(1+2) \ldots . . v(i+m-1)]
\end{aligned}
$$

Siendo el número de vectores $\mathrm{y}_{\mathrm{m}}$ (j) cuya distancia de $\mathrm{x}_{\mathrm{m}}$ (j) se define como:

$d\left[x_{m}(i), y_{m}(i)\right]=\max \{|u(i+k)-v(j+k)|: 0 \leq k \leq \cdots m-1\}$

Correspondiente a una tolerancia $r$. Similarmente, $\mathrm{n}_{\mathrm{i}}^{(\mathrm{m}+1)}$ es el número de coincidencias de longitud $\mathrm{m}+1$. Finalmente, la Cross-SampEn es calculada así:

$$
\begin{aligned}
& \text { cross-SampEn }(\mathrm{u}, \mathrm{v}, \mathrm{m}, \mathrm{r})=\cdots \\
& -\ln \left(\sum_{\mathrm{i}=1}^{\mathrm{N}-\mathrm{m}} \mathrm{n}_{\mathrm{i}}^{(\mathrm{m}+1)} / \sum_{\mathrm{i}=1}^{\mathrm{N}-\mathrm{m}} \mathrm{n}_{\mathrm{i}}^{(\mathrm{m})}\right)
\end{aligned}
$$

Es habitual que las dos series de tiempo se normalicen primero, al restar el valor medio de cada serie de datos y luego dividiéndolas entre la desviación estándar. Esta normalización es válida, ya que el interés principal es comparar patrones ${ }^{[19]}$.

\section{Entropía condicional}

La entropía condicional (Conditional entropy, Cond $H$ ) corresponde a la incertidumbre que permanece en una variable cuando otra variable tiene un valor conocido ${ }^{[20]}$. Está delimitada por 0 y por la entropía de la variable original. El límite inferior es verdadero cuando $X$ es completamente dependiente de $Y$, y el límite superior es verdadero cuando $X$ e $Y$ son independientes entre sí (Ecuaciones 7 y 8) ${ }^{[20]}$.

$$
\begin{gathered}
H(X \mid Y)=\sum_{j=1}^{n} p\left(y_{j}\right) H\left(X \mid Y=y_{j}\right) \\
0 \leq h(X \mid Y) \leq H(x)
\end{gathered}
$$

Donde la entropía dependiente $H(X \mid Y)$ corresponde a la dependencia de $X$ en $Y$ o viceversa dentro de las probabilidades de $x$ e $y(p(x), p(y))$. Donde $X$ es una variable aleatoria con $n$ valores posibles, $H(X)$ y $H(Y)$ corresponden a las entropías de $X$ e $Y$. Adicionalmente, se observa que la $C o n d H$ es igual a la entropía conjunta menos la entropía de la variable condicional (Ecuación 9).

$$
H(X \mid Y)=H(X Y)-H(Y)
$$

Por lo tanto, si la CondH es más cercana a la entropía de la variable $+H(x)$, el acoplamiento CRP será mayor y si CondH tiende a 0 el acoplamiento CRP será menor.

\section{Información mutua}

La información mutua (Mutual information, MI) se define como una medida cuánta información de una variable determina la información de otra variable ${ }^{[20]}$. La información mutua se puede definir de dos maneras diferentes pero equivalentes, ya sea en términos de entropías o en términos de distribuciones de probabilidad, y es simétrica con respecto al orden de X y Y [20] (ecuaciones 10, 11 y 12).

$$
\begin{aligned}
& I(X ; Y)=H(X)-H(X \mid Y) \\
& I(X ; Y)=H(Y)-H(Y \mid X)
\end{aligned}
$$

$$
I(X ; Y)=H(X)+H(Y)-H(X, Y)
$$

La información mutua se entiende como la superposición entre las variables y, por lo tanto, se puede obtener como la suma de las dos entropías $\mathrm{H}(\mathrm{X}), \mathrm{H}(\mathrm{Y})$, menos la entropía conjunta $\mathrm{H}(\mathrm{X}, \mathrm{Y})$; lo cual elimina toda la información mutua que no está contenida en las variables ${ }^{[20]}$. 
De ello se puede inferir que, si la $M I$ tiende a 0 , hay menor asociación entre variables; por el contrario, si la $M I$ tiende a un valor positivo de $\mathrm{X}$ o $\mathrm{Y}$ como resultado de la resta de $\mathrm{H}(\mathrm{X} ; \mathrm{Y})-\mathrm{H}(\mathrm{X} \mid \mathrm{Y})$, la asociación es mayor.

\section{Información m utua de Rényi}

La información mutua de Rényi (Rényi’s Mutual Information, RényiMI) es definida como la divergencia entre la densidad de probabilidad conjunta y el producto de las marginales. En general, RényiMI es definida como $\mathrm{I}_{\alpha}(\mathrm{X} ; \mathrm{Y})^{[20]}$; y la divergencia de Rényi como:

$$
\begin{gathered}
\mathrm{D}_{\alpha}(\mathrm{p}(\mathrm{x}, \mathrm{y})) \| \mathrm{p}(\mathrm{x}) \mathrm{p}(\mathrm{y})= \\
\ldots \frac{1}{\alpha-1} \log \sum_{\mathrm{x} \in \mathrm{X}} \sum_{\mathrm{y} \in \mathrm{X}} \mathrm{p}(\mathrm{x}, \mathrm{y})^{\alpha} \mathrm{p}(\mathrm{x})^{1-\alpha} \mathrm{p}(\mathrm{y})^{1-\alpha} \\
\text { Cuando } \alpha \rightarrow 1 \\
\lim _{\alpha \rightarrow 1} \mathrm{I}_{\alpha}(\mathrm{X} ; \mathrm{Y}) .=\mathrm{I}(\mathrm{X} ; \mathrm{Y})
\end{gathered}
$$

Donde $\alpha$ es utilizado para hallar la divergencia entre dos distribuciones de probabilidad; y se define: $\forall \alpha \neq 1$.

La RényiMI cuantifica la cantidad de información compartida entre dos variables con valor alto cuando las variables están fuertemente correlacionadas; y con valor bajo cuando son fuertemente independientes ${ }^{[20]}$.

\section{Análisis estadístico}

Los datos fueron analizados para determinar la normalidad de los grupos mediante la prueba de Kolmogorov-Smirnov, utilizando el software estadístico GraphPad Prism (San Diego, California, EE.UU.).

Se aplicaron comparaciones entre todos los grupos mediante ANOVA de dos vías, seguida de una prueba de Fisher LSD post-hoc. Los datos se expresaron como promedio \pm EEM y el nivel de significancia se estableció en $\alpha=0.05$. Finalmente se compararon los niveles de probabilidad (p) para establecer el algoritmo que mejor detectara la presencia o ausencia del desacoplamiento CRP debido a la endotoxemia.

\section{RESULTADOS}

En la Figura 1 se pueden observar con gráficas de barras los resultados del análisis del acoplamiento CRP de los cinco métodos utilizados:

a)

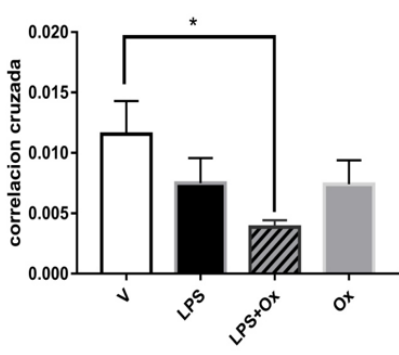

c)

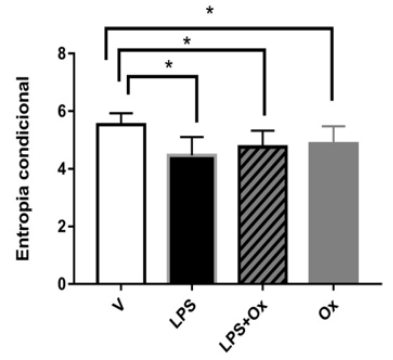

b)

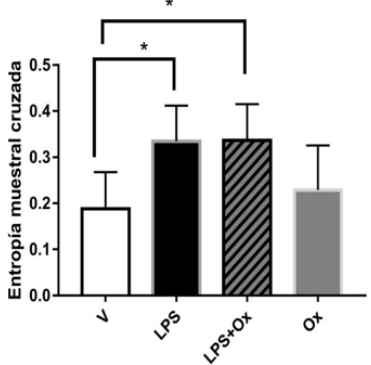

d)

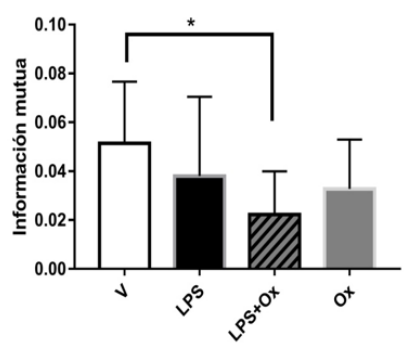

e)

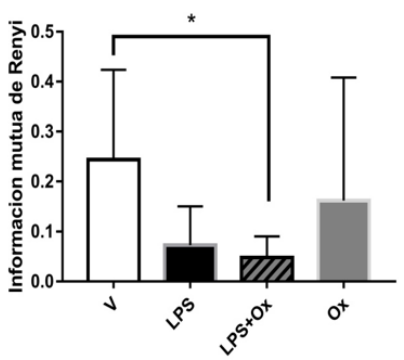

FIGURA 1. Gráficas de barras para los cuatro grupos de estudio en el largo plazo $+7 \mathrm{~h}$ : Vehículo (V), Lipopolisacárido (LPS), Lipopolisacárido + Oxitocina (LPS + Ox) y Oxitocina (Ox). En los diferentes paneles se muestran los resultados de los diferentes análisis para cuantificar el acoplamiento cardiorrespiratorio: a) correlación cruzada (X-Corr) b) entropía muestral cruzada (Cross-SampEn) c) entropía condicional (CondH) d) información mutua (MI) y e) información mutua de Renyi (RényiMI), * significancia estadística entre grupos donde $\mathrm{p}<0.05$. 
1a) correlación cruzada (X-Corr); 1b) entropía muestral cruzada (Cross-SampEn); 1c) entropía condicional (CondH); 1d) información mutua (MI) y 1e) información mutua de Rényi (RényiMI).

La Figura 1a muestra los resultados de la $X$-Corr, en los cuales se puede notar una disminución en su valor en el grupo LPS + Ox en comparación con V, el cual fue estadísticamente significativo de acuerdo con la prueba ANOVA ( $\mathrm{F}=2.467 ; \mathrm{p}=0.0120)$. Adicionalmente, los resultados de la Cross-SampEn mostrados en la Figura $1 \mathrm{~b}$ muestran diferencias significativas $(\mathrm{F}=$ 5.676) entre los grupos V vs LPS ( $\mathrm{p}=0.0031), \mathrm{V}$ vs LPS + Ox ( $\mathrm{p}=0.0029)$ y LPS vs Ox $(\mathrm{p}=0.0260)$. Por otro lado, la Cond $H$ arrojó diferencias significativas $(F=4.489)$ entre los otros grupos: V vs LPS $(\mathrm{p}=0.0016), \mathrm{V}$ vs LPS + Ox $(\mathrm{p}=0.0168)$ y V vs Ox ( $\mathrm{p}=0.0375)$, Fig. 1c.
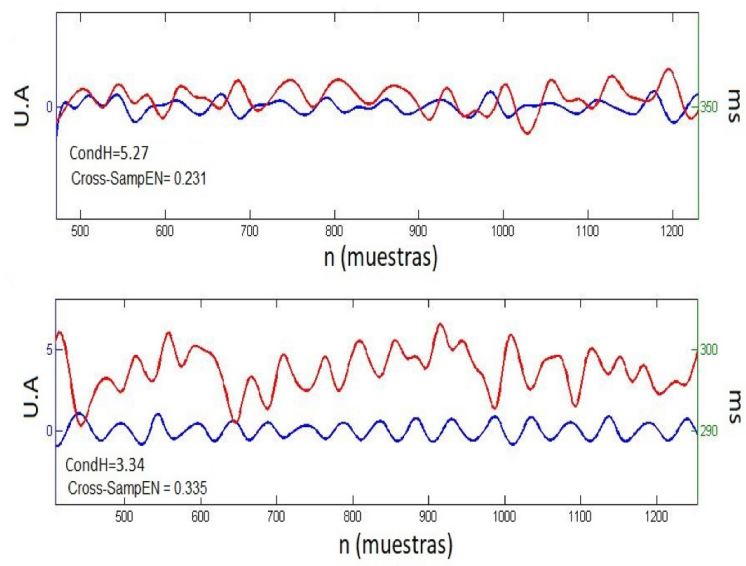

FIGURA 2. Señales representativas de las series de tiempo

$\mathbf{R}-\mathbf{R}$ (rojo) con unidades en ms y señales respiratorias derivadas del electrocardiograma EDR (azul) con unidades arbitrarias (U.A.) interpoladas con un spline cúbico a 100 Hz. De manera visual se aprecia un mayor acoplamiento cardiorrespiratorio en el inciso a) roedor del grupo vehículo a las $+7 \mathrm{~h}$ posteriores al tratamiento ( $V$, solución salina) en comparación con b) roedor del grupo lipopolisacárido a las +7 $\mathrm{h}$ posteriores al tratamiento (LPS). En el marco inferior izquierdo se indican los valores de entropía condicional (CondH) y entropía muestral cruzada (Cross-SampEn) como indicadores del acoplamiento cardiorrespiratorio (CRP).
La Figura 1d exhibe los resultados del método de MI; los grupos que tuvieron diferencias significativas ( $\mathrm{F}=$ 1.71) fueron únicamente $V$ vs LPS $+O x(p=0.0358)$. Finalmente, la Figura 1e revela los resultados de la RényiMI, en donde los grupos con diferencias estadísticas $(\mathrm{F}=2.219)$ fueron V vs LPS + Ox $(\mathrm{p}=0.0300)$.

La Figura 2a muestra señales representativas de EDR y R-R de una rata del grupo $\mathrm{V}$ a las $+7 \mathrm{~h}$, por otro lado, la Figura 2b muestra de igual manera las señales EDR y R-R de una rata del grupo LPS a las $+7 \mathrm{~h}$. De manera visual se puede apreciar la presencia de un mayor acoplamiento CRP en la rata del grupo V en comparación con la rata del grupo LPS.

\section{DISCUSIÓN}

Este es uno de los primeros estudios que comparó diversos métodos lineales y no lineales para evaluar los efectos de la oxitocina en el acoplamiento CRP durante la endotoxemia. El acoplamiento CRP se asocia a la sinergia entre los sistemas cardiovascular y respiratorio. Estudios indican que esta interacción se origina en el tronco cerebral y las neuronas cardiacas vágales del SNA ${ }^{[21]}$. Dichas estructuras cerebrales son esenciales para el control de la respiración y del ritmo cardiaco.

Adicionalmente, se ha demostrado que la manifestación de ciertas patologías como las apneas de prematuridad, el síndrome de muerte súbita del lactante, la apnea obstructiva del sueño y el síndrome de Rett favorecen el desacoplamiento CRP, lo cual estaría ligado a una presunta disautonomía en las funciones reguladoras de los sistemas cardiaco y respiratorio ${ }^{[21]}$. Otros hallazgos sobre endotoxemia bacteriana inducida por LPS en mamíferos indican que el LPS provoca un cuadro de inflamación sistémico y de estrés biológico que afecta a múltiples sistemas corporales como lo son el cardiaco, respiratorio, renal y termorregulador, lo cual origina ajustes en las frecuencias cardiaca y respiratoria, vasodilatación cutánea, aumento de la temperatura, entre otras ${ }^{[11,13,22,23]}$. 
De acuerdo con la literatura, los valores de los métodos $X$-Corr ${ }^{[24]}$, CondH ${ }^{[25]}, M I^{[24]}$ y RényiMI ${ }^{[26]}$ al ser más cercanos al valor de cero $(\mathrm{R} \rightarrow 0)$ son indicativos de un menor acoplamiento entre dos series de tiempo. Contrariamente, valores elevados de la Cross-SampEn [8] $(\mathrm{R} \rightarrow+\infty)$ sugieren un desacoplamiento entre dos series de tiempo. Nuestros resultados muestran que entre los métodos utilizados en este estudio para cuantificar el desacoplamiento CRP, los métodos de CrossSampEn (fig. 1b) y CondH (fig. 1c) fueron los que presentaron mayores diferencias significativas $(\mathrm{p}<0.01)$. Específicamente, los valores altos de Cross-SampEn y bajos de CondH están asociados a la existencia de un desacoplamiento CRP en los grupos LPS vs V, LPS + Ox, y Ox vs V, respectivamente. De manera interesante, los métodos de X-Corr, MI y RényiMI no detectaron el desacoplamiento CRP entre V vs LPS, únicamente lo hicieron para $\mathrm{V}$ vs. LPS+Ox.

Los hallazgos del presente estudio indican que las fluctuaciones cardiacas y respiratorias están desacopladas durante el periodo de endotoxemia, esto sugiere que las funciones reguladoras de estos sistemas se ven modificadas debido al proceso de inflamación sistémico que desencadena el LPS ${ }^{[11,13]}$. También se encontró que el grupo LPS + Ox tuvo un menor acoplamiento CRP en comparación con el grupo V (fig. 1a; fig. 1d; fig. 1e), lo que sugiere que la oxitocina no posee efectos fisiológicos que favorezcan el acoplamiento CRP ${ }^{\text {[27]. }}$

Estudios revelan que la oxitocina es un neuropéptido que provoca excitación en el sistema arterial y endocrino, lo que podría provocar de manera indirecta un aumento de la frecuencia respiratoria ${ }^{[28]}$. En contraste, nosotros no encontramos diferencias significativas en la frecuencia cardiaca y respiratoria en roedores tratados únicamente con oxitocina en nuestros estudios previos ${ }^{[11,13]}$. Las investigaciones apuntan a que la oxitocina en ratas activa los receptores neuronales de esta hormona provocando cambios en los parámetros cardiorrespiratorios ${ }^{[29]}$. En este sentido, una alteración en la frecuencia cardiaca o respiratoria debido a la oxitocina podría promover el desacoplamiento CRP (fig 1c). En nuestros estudios previos encontramos que la oxitocina parece actuar como un péptido cardioprotector al favorecer el acoplamiento autonómico colinérgico cardíaco durante la endotoxemia ${ }^{[13]}$. Dicho efecto cardioprotector se origina debido a que la oxitocina estimula la secreción de péptido natriurético atrial, el cual tiene efectos cardioprotectores comprobados ${ }^{[30]}$. A pesar de los efectos comprobados de la oxitocina como agente cardioprotector y antiinflamatorio, los hallazgos del presente trabajo indican que no parece restablecer el acoplamiento CRP durante endotoxemia.

Finalmente, la Figura 2 muestra las señales EDR y R-R de una rata del grupo V y del grupo LPS a las $+7 \mathrm{~h}$. De manera visual se puede apreciar un mayor acoplamiento CRP en el grupo V (Fig 2a) en comparación con LPS (Fig 2b). Dicha apreciación visual es concordante con los valores de los métodos CondH y Cross-SampEn. Estos datos sugieren que existe un desacoplamiento CRP producido por la endotoxemia inducida por LPS a largo plazo y que todos los métodos explorados aquí son capaces de detectarlo. Otros hallazgos señalan que la frecuencia cardiaca y la respiratoria están altamente ligadas, por lo cual el cambio en la frecuencia de ventilación o del ritmo cardiaco afecta el ritmo cardiorrespiratorio, estas fluctuaciones se ven mayormente afectadas en procesos patológicos que alteran la actividad nerviosa vagal ${ }^{[4,31]}$, como ejemplo se presenta la endotoxemia inducida por LPS.

Es importante mencionar que la aportación adicional de los resultados aquí presentados respecto a trabajos previos ${ }^{[13]}$, se basan en la implementación de métodos lineales y no lineales para evaluar el desacoplamiento CRP durante la endotoxemia inducida por LPS y los efectos de la oxitocina sobre este. Entre los métodos no lineales se encontró que CondH y Cross-SampEn tuvieron los valores más bajos de (p) entre el grupo V vs LPS en comparación con los métodos MI y RényiMI e 
incluso el método lineal de $X$-Corr. Esto puede deberse a que tanto CondH y Cross-SampEn están basados en el análisis de la entropía, el cual ya ha sido utilizado para evaluar satisfactoriamente el acoplamiento CRP en jóvenes y adultos de edad media ${ }^{[1]} \mathrm{y}$ recientemente en estados de depresión ${ }^{[32]}$.

\section{LIMITACIONES}

Los algoritmos computacionales utilizados fueron seleccionados por su robustez y validez para la detección de acoplamientos entre series de tiempo; sin embargo, existen otros métodos novedosos que podrían complementar nuestros resultados. Por ejemplo, se ha explorado el método de análisis simbólico conjunto para cuantificar el acoplamiento CRP entre las series de tiempo R-R y señales respiratorias ${ }^{[33]}$. En estudios futuros se implementarán nuevas metodologías para la cuantificación del acoplamiento CRP.

Por otro lado, la dosis y la vía de administración de la oxitocina (subcutánea) podrían no ser las indicadas para revelar sus efectos en el acoplamiento CRP, a pesar que en nuestros estudios previos fue capaz de demostrar efectos cardioprotectores durante la endotoxemia inducida por LPS ${ }^{[11]}$. En estudios futuros es deseable incrementar la dosis de oxitocina y probar nuevas vías de administración, como es el caso de la vía intracerebroventricular (ICV), ya que se ha demostrado que esta administración intracerebral produce cambios en la frecuencia cardiaca ${ }^{[34]}$. La vía de administración la dosis podrían marcar una diferencia en los efectos de la oxitocina en el acoplamiento CRP.

\section{CONCLUSIONES}

En el presente estudio encontramos como hallazgo principal que los métodos de Cross-SampEn y CondH fueron los que presentaron mayores diferencias estadísticas para identificar el desacoplamiento CRP. Adicionalmente se encontró que el desacoplamiento CRP en ratas se ve aumentado por la endotoxemia inducida por LPS en el largo plazo y que la oxitocina administrada subcutáneamente no parece disminuir el desacoplamiento CRP durante la endotoxemia. En conclusión, los métodos no lineales (CondH y CrossSampEn) parecen ser más apropiados para detectar el desacoplamiento CRP durante la endotoxemia inducida por LPS en comparación con la correlación cruzada. Por lo cual podrían ser una herramienta prometedora para el diagnóstico/pronóstico de procesos inflamatorios sistémicos. 


\section{REFERENCIAS}

[1] Kapidžić A, Platiša MM, Bojić T, Kalauzi A. Nonlinear properties of cardiac rhythm and respiratory signal under paced breathing in young and middle-aged healthy subjects. Med Eng Phys [Internet]. 2014 Dec;36(12):1577-84. Available from: https://linkinghub. elsevier.com/retrieve/pii/S135045331400215X

[2] ChuDuc H, NguyenPhan K, NguyenViet D. A Review of Heart Rate Variability and its Applications. APCBEE Procedia [Internet]. 2013;7:80-5. Available from: https://linkinghub.elsevier.com/ retrieve/pii/S2212670813001188

[3] Toledo E, Akselrod S, Pinhas I, Aravot D. Does synchronization reflect a true interaction in the cardiorespiratory system? Med Eng Phys [Internet]. 2002 Jan;24(1):45-52. Available from: http:// linkinghub.elsevier.com/retrieve/pii/S135045330100114X

[4] Penzel T, Kantelhardt JW, Bartsch RP, Riedl M, Kraemer JF, Wessel $\mathrm{N}$, et al. Modulations of Heart Rate, ECG, and Cardio-Respiratory Coupling Observed in Polysomnography. Front Physiol [Internet]. 2016 Oct 25;7(October). Available from: http://journal.frontiersin. org/article/10.3389/fphys.2016.00460/full

[5] Berger S, Kliem A, Yeragani V, Bär KJ. Cardio-respiratory coupling in untreated patients with major depression. J Affect Disord. 2012;139(2):166-71.

[6] Riedl M, Müller A, Kraemer JF, Penzel T, Kurths J, Wessel N. Cardio-respiratory coordination increases during sleep apnea. PLoS One. 2014;9(4).

[7] Thomas RJ, Weiss MD, Mietus JE. Prevalent hypertension and stroke in the sleep heart health study: association with an ECGderived spectrographic marker of cardiopulmonary coupling. Sleep. 2009;

[8] Chang JS, Lee SD, Ju G, Kim J-W, Ha K, Yoon I-Y. Enhanced cardiorespiratory coupling in patients with obstructive sleep apnea following continuous positive airway pressure treatment. Sleep Med [Internet]. 2013 Nov;14(11):1132-8. Available from: http://dx.doi.org/10.1016/j.sleep.2013.04.02

[9] Dick TE, Morris KF. Quantitative analysis of cardiovascular modulation in respiratory neural activity. J Physiol [Internet]. 2004 May;556(3):959-70. Available from: http://doi.wiley.com/10.1113/jphysiol.2003.060418

[10] Gholami M, Mazaheri P, Mohamadi A, Dehpour T, Safari F, Hajizadeh S, et al. Endotoxemia is Associated With Partial Uncoupling of Cardiac Pacemaker From Cholinergic Neural Control in Rats. Shock [Internet]. 2012 Feb;37(2):219-27. Available from: http://content.wkhealth.com/linkback/openurl?sid=WKPTLP:landing page \&an $=00024382-201202000-00015$

[11] Reyes-Lagos JJ, Hadamitzky M, Peña-Castillo MÁ, Echeverría JC, Bösche K, Lückemann L, et al. Exogenous oxytocin reduces signs of sickness behavior and modifies heart rate fluctuations of endotoxemic rats. Physiol Behav [Internet]. 2016 Oct;165:223-30. Available from: http://dx.doi.org/10.1016/j.physbeh.2016.07.013

[12] Gamer M, Büchel C. Oxytocin specifically enhances valencedependent parasympathetic responses. Psychoneuroendocrinology [Internet]. 2012 Jan;37(1):87-93. Available from: https://linkinghub. elsevier.com/retrieve/pii/S0306453011001600
[13] Elorza-Ávila AR, Reyes-Lagos JJ, Hadamitzky M, Peña-Castillo MÁ, Echeverría JC, Ortiz-Pedroza M del R, et al. Oxytocin's role on the cardiorespiratory activity of endotoxemic rats. Respir Physiol Neurobiol [Internet]. 2017 Feb;236:19-22. Available from: https:// linkinghub.elsevier.com/retrieve/pii/S156990481630163X

[14] Silva I, Moody GB. An Open-source Toolbox for Analysing and Processing PhysioNet Databases in MATLAB and Octave. J Open Res Softw [Internet]. 2014 Sep 24;2. Available from: http://doi.org/10.5334/jors.bi

[15] Gavin Brown, Adam Pocock, Ming-Jie Zhao ML. Conditional Likelihood Maximisation: A Unifying Framework for Information Theoretic Feature Selection. J Mach Learn Res. 2016;53:46-58.

[16] Yaghmaie N, Maddah-Ali MA, Jelinek HF, Mazrbanrad F. Dynamic signal quality index for electrocardiograms. Physiol Meas [Internet]. 2018 Oct 22;39(10):105008. Available from: http://stacks. iop.org/0967-3334/39/i=10/a=105008?key=crossref.9737bac99baf33f a220f4d9156db4de1

[17] Pincus SM. Approximate entropy as a measure of irregularity for psychiatric serial metrics. Bipolar Disord [Internet]. 2006 Oct;8(5p1):430-40. Available from: http://doi.wiley.com/10.1111/j.1399-5618.2006.00375.x

[18] Chang JS, Ha K, Yoon I-Y, Yoo CS, Yi SH, Her JY, et al. Patterns of cardiorespiratory coordination in young women with recurrent major depressive disorder treated with escitalopram or venlafaxine. Prog Neuro-Psychopharmacology Biol Psychiatry [Internet]. 2012 Oct;39(1):136-42. Available from: https:// linkinghub.elsevier.com/retrieve/pii/So278584612001376

[19] Yin Y, Shang P, Feng G. Modified multiscale cross-sample entropy for complex time series. Appl Math Comput [Internet]. 2016 Oct;289:98-110. Available from: https://linkinghub.elsevier.com/ retrieve/pii/Soog6300316303204

[20] Pocock A. MSc Project Feature Selection using Information Theoretic Techniques. Computer (Long Beach Calif). 2008;

[21] Garcia AJ, Koschnitzky JE, Dashevskiy T, Ramirez J-M. Cardiorespiratory coupling in health and disease. Auton Neurosci [Internet]. 2013 Apr;175(1-2):26-37. Available from: http://dx.doi.org/10.1016/j.autneu.2013.02.006

[22] Cazzola M, Calzetta L, Page CP, Rinaldi B, Capuano A, Matera MG. Protein Prenylation Contributes to the Effects of LPS on EFSInduced Responses in Human Isolated Bronchi. Am J Respir Cell Mol Biol [Internet]. 2011 Oct;45(4):704-10. Available from: https://doi.org/10.1165/rcmb.2010-03060C

[23] McDonald FB, Chandrasekharan K, Wilson RJA, Hasan SU. Cardiorespiratory control and cytokine profile in response to heat stress, hypoxia, and lipopolysaccharide (LPS) exposure during early neonatal period. Physiol Rep [Internet]. 2016 Jan;4(2):e12688. Available from: http://doi.wiley.com/10.14814/phy2.12688

[24] Hoyer D, Leder U, Hoyer H, Pompe B, Sommer M, Zwiener U. Mutual information and phase dependencies: measures of reduced nonlinear cardiorespiratory interactions after myocardial infarction. Med Eng Phys [Internet]. 2002 Jan;24(1):33-43. Available from: http://linkinghub.elsevier.com/retrieve/pii/ S1350453301001205 
[25] Faes L, Nollo G, Porta A. Non-uniform multivariate embedding to assess the information transfer in cardiovascular and cardiorespiratory variability series. Comput Biol Med [Internet]. 2012 Mar;42(3):290-7. Available from:

http://dx.doi.org/10.1016/j.compbiomed.2011.02.007

[26] Kopylova Y, Buell DA, Huang C-T, Janies J. Mutual information applied to anomaly detection. J Commun Networks [Internet]. 2008 Mar;10(1):89-97. Available from: http://ieeexplore.ieee.org/ document/6388332/

[27] Mack SO, Kc P, Wu M, Coleman BR, Tolentino-Silva FP, Haxhiu MA. Paraventricular oxytocin neurons are involved in neural modulation of breathing. J Appl Physiol [Internet]. 2002 Feb;92(2):826-34. Available from: https://doi.org/10.1152/japplphysiol.00839.2001

[28] Henry JL, Sessle BJ. Vasopressin and oxytocin express excitatory effects on respiratory and respiration-related neurones in the nuclei of the tractus solitarius in the cat. Brain Res [Internet]. 1989 Jul;491(1):150-5. Available from: http://linkinghub.elsevier.com/ retrieve/pii/0006899389900978

[29] Granjeiro ÉM, Marroni SS, Martins Dias DP, Heck Bonagamba LG, Costa KM, dos Santos JC, et al. Behavioral and Cardiorespiratory Responses to Bilateral Microinjections of Oxytocin into the Central Nucleus of Amygdala of Wistar Rats, an Experimental Model of Compulsion. Ashton N, editor. PLoS One [Internet]. $2014 \mathrm{Jul}$ 18;9(7):e99284. Available from: https://dx.plos.org/10.1371/journal.pone.0099284
[30] Gutkowska J, Jankowski M, Antunes-Rodrigues J. The role of oxytocin in cardiovascular regulation. Brazilian J Med Biol Res. 2014;47(3):206-14.

[31] Yasuma F, Hayano J. Respiratory Sinus Arrhythmia. Chest [Internet]. $2004 \mathrm{Feb} ; 125(2): 683-90$. Available from: http://dx.doi.org/10.1378/chest.125.2.683

[32] Zhao L, Yang L, Su Z, Liu C. Cardiorespiratory Coupling Analysis Based on Entropy and Cross-Entropy in Distinguishing Different Depression Stages. Front Physiol [Internet]. 2019 Mar 29;10:1-14. Available from: https://doi.org/10.3389/fphys.2019.00359

[33] Baumert M, Javorka M, Kabir M. Joint symbolic dynamics for the assessment of cardiovascular and cardiorespiratory interactions. Philos Trans R Soc A Math Phys Eng Sci [Internet]. 2014 Dec 29;373(2034):20140097-20140097. Available from: https://doi.org/10.1098/rsta.2014.0097

[34] Petersson M, Uvnäs-Moberg K. Effects of an acute stressor on blood pressure and heart rate in rats pretreated with intracerebroventricular oxytocin injections. Psychoneuroendocrinology [Internet]. 2007 Sep;32(8-10):959-65. Available from: https://linkinghub.elsevier.com/retrieve/pii/ S0306453007001667 